\title{
Does Pomegranate intake attenuate cardiovascular risk factors in hemodialysis patients?
}

Lilach Shema-Didi $^{1 \dagger}$, Batya Kristal ${ }^{2,3+}$, Shifra Sela ${ }^{3,4,5}$, Ronit Geron ${ }^{2}$ and Liora Ore $5^{5^{*}}$

\begin{abstract}
Background: Atherosclerotic cardiovascular disease (CVD) is the most common cause of morbidity and mortality among hemodialysis (HD) patients. It has been attributed, among other causes, to hypertension and dyslipidemia. The aim of the present study was to investigate the effect of a year-long consumption of Pomegranate juice (PJ), on two traditional cardiovascular (CV) risk factors: hypertension and lipid profile, as well as on cardiovascular events.

Methods: $101 \mathrm{HD}$ patients were randomized to receive 100 cc of PJ (0.7 mM polyphenols) or matching placebo juice, three times a week for one year. The primary endpoints were traditional $\mathrm{CV}$ risk factors; blood pressure and lipid profile. Systolic, diastolic and pulse pressure, plasma levels of triglycerides (TG), high density lipoprotein (HDL), low density lipoprotein (LDL) and total cholesterol were monitored quarterly during the study year. Secondary endpoint was incidence of cardiovascular events.
\end{abstract}

Results: PJ consumption yielded a significant time response improvement in systolic blood pressure, pulse pressure, triglycerides and HDL level; an improvement that was not observed in the placebo intake group. These beneficial outcomes were more pronounced among patients with hypertension, high level of triglycerides and low levels of HDL.

Conclusion: Regular PJ consumption by HD patients reduced systolic blood pressure and improved lipid profile. These favorable changes may reduce the accelerated atherosclerosis and high incidence of CVD among HD patients.

Trial registration: ClinicalTrials.gov registry, Identifier number: NCT00727519

Keyword: Pomegranate juice hemodialysis, Polyphenols, Hypertension, Lipid profile

\section{Background}

Patients on renal replacement therapy (RRT) are at increased risk of cardiovascular (CV) mortality and morbidity compared to the general population [1]. Every year, between $10-20 \%$ of all patients on dialysis die, with about $45 \%$ of deaths attributed to CV causes [2]. Established 'traditional' atherosclerosis risk factors, such as hypertension and dyslipidemia, have been recognized as independent predictors of cardiovascular disease (CVD) among chronic kidney disease (CKD) [1] and hemodialysis (HD)

\footnotetext{
* Correspondence: liora.ore@gmail.com

${ }^{\dagger}$ Equal contributors

${ }^{5}$ School of Public Health, Faculty of Social Welfare \& Health Sciences,

University of Haifa, Haifa, Israel

Full list of author information is available at the end of the article
}

patients $[3,4]$. Blood pressure is commonly high in HD patients. This phenomenon has been attributed to several causes, among them the chronic volume overload in HD patients, due to impaired blood pressure homoeostasis function [4]. In addition to the high prevalence of hypertension, HD patients usually display elevated triglycerides (TG), reduced high density lipoprotein (HDL) cholesterol and elevated concentration of lipoprotein-a [3,5], while total and low density lipoprotein (LDL) cholesterol usually remain within normal limits $[5,6]$. Several clinical trials and meta-analyses have shown the cardiovascular benefits of lowering blood pressure in patients with kidney disease

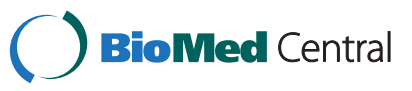

(C) 2014 Shema-Didi et al.; licensee BioMed Central Ltd. This is an Open Access article distributed under the terms of the Creative Commons Attribution License (http://creativecommons.org/licenses/by/2.0), which permits unrestricted use, distribution, and reproduction in any medium, provided the original work is properly credited. 
$[7,8]$ and patients on dialysis [4]. Although the cardiovascular benefits of improving lipid profile among dialysis patients is controversial [9], there is evidence that treatment of HD patients with lipid lowering drugs is associated with reduced CV mortality [10]. Therefore, improving lipid profile and reduction of blood pressure is a therapeutic target for patients on chronic dialysis.

It has been known for many years that high intake of fruits and vegetables is associated with reduced risk of coronary heart disease [11]. The beneficial effect of fruits and vegetables may be related especially to flavonoids, which are thought to exert their action by inhibiting LDL oxidation and platelet aggregation [12], as well as to inhibit the angiotensin converting enzyme (ACE), a key component in the renin angiotensin aldosterone system (RAAS) which regulates blood pressure [13]. Pomegranate juice (PJ) is a rich source of flavonoids and as such it has potent antioxidant activity. The flavonoids it contains have been linked to a diverse group of polyphenols, including ellagitanins, gallotannins and ellegic acid.

PJ antioxidant activity was studied mainly with regard to cardiovascular function among non HD patients. Different studies demonstrated the anti-atherogenicity properties of PJ by its ability to lower serum angiotensin converting enzyme (ACE) activity which resulted in systolic blood pressure reduction [13], decreased common carotid artery intima-media thickness (IMT) [14] and attenuation of the myocardial ischemia in patients who had congestive heart disease [15]. Recently, studies suggested that PJ consumption may be beneficial in populations at high risk to develop atherosclerosis and CVD $[16,17]$. The antioxidative effects of PJ were more impressive in diabetic patients than in healthy controls [17], leading to the assumption that PJ may have beneficial effect in patients exposed to oxidative stress (OS) burden. Since HD patients are exposed to the most severe systemic OS compared to other clinical states, PJ intake in this high risk population may be more effective than in other groups of patients. We have shown the beneficial effects of consistent consumption over one year's time of PJ on 'non traditional' CV risk factors, such as OS and inflammation, and on clinical outcome such as reduction in intima media thickness [18]. Furthermore, we have demonstrated PJ's ability to reduce the incidence of infections, which is the second most common cause of morbidity and mortality of HD patients [18] However, the effect of PJ on 'traditional' risk factors, such as hypertension and lipid profile has not yet been studied among HD patients.

The present study aims to characterize for the first time, the long term effects of PJ consumption by HD patients on hypertension, lipid profile and incidence of CVD.

\section{Methods}

\section{Study population}

One dialysis center at the Western Galilee Hospital, Nahariya, Israel participated in the study. Eligible participants were chronic HD patients aged $>18$ years who underwent $3 \mathrm{~h}$ HD sessions weekly using low-flux high performance cellulose-triacetate [sureflux-190G, NIPRO] or polysulfone membranes [FX 10, Fresenius]. Exclusion criteria included: HD treatment for less than 3 months, simultaneous participation in other clinical trials, patient's refusal or inability to give informed consent due to mental or physical state, or pregnancy (actual or planned) during study period. One hundred and forty-nine HD patients were identified as eligible. Of these, $101 \mathrm{HD}$ patients were recruited (detailed information regarding the study population was published in our previous paper [18]). Recruited patients did not differ from eligible subjects in demographic, dialysis and comorbidity characteristics [18]. All individuals gave their written informed consent to participate in the study. The study was approved by the Institutional Ethics Committee at the study center (Helsinki Committee approval no. 2008-06-06). The study was registered in ClinicalTrials.gov registration, Identifier number: NCT00727519.

\section{Study design}

The study was a double-blind, placebo-controlled, randomized, clinical trial. Two groups of HD patients were compared: one group $(n=66)$ received $0.7 \mathrm{mmol}$ of polyphenols in the form of $100 \mathrm{cc}$ of PJ (Naturafood) and the other $(n=35)$ received a matching $100 \mathrm{cc}$ placebo juice three times a week during the first dialysis treatment hour, for one year. Patients, medical and laboratory staff were all blinded to patient's group allocation. The smaller randomization ratio of $2 ; 1$, with only a modest loss in statistical power [19] was chosen due to ethical (a potential PJ effect according to previous studies) and feasibility considerations. The study sample size $(n=101)$, with a ratio of 2:1, PJ: Placebo, was calculated to have $80 \%$ power $(\mathrm{p}=$ 0.05 ) to detect $5 \%$ decrease in SBP (as demonstrated previously among hypertensive patients [13]), assuming a mean SBP level of $140 \pm 20 \mathrm{~mm} \mathrm{Hg}$ according to our preliminary results.

During the study period patients were instructed not to drink any other fresh fruit juice at home. Verification of juice intake was carried out by the study investigator and was documented. Patients who did not drink the juice at least 3 times were excluded from the study. Blood for lipid profile measurements was drawn at 0, 3, 6 and 12 months of study intervention, always before dialysis, from the arterial sampling port closest to the patient. Three sequential blood pressure (BP) measurements performed before dialysis initiation were used to calculate the mean systolic and diastolic blood pressure 
(SBP, DBP) at 0, 3, 6 and 12 months of study intervention. Pulse pressure (PP) was calculated by SBP minus DBP.

\section{PJ Juice}

In order to choose the commercial PJ with the highest polyphenols levels, several hand squeezed and different commercial juices available in Israel were analyzed by the colorimetric assay in Migal Galilee Technology Center, as previously described $[20,21]$. PJ from Naturafood manufacturer [Turkey] was found to have the highest concentration of polyphenols, each $100 \mathrm{cc}$ of PJ contained $0.7 \mathrm{mmol}$ of polyphenols, and was used in this study. The juice was kept at room temperature $<25^{\circ} \mathrm{C}$ until used.

\section{Placebo Juice}

The placebo was chosen to resemble the pomegranate juice in color and taste and was prepared especially for this study by food engineers. The placebo contained: pomegranate artificial extract of Frutarom Ltd; Citric acid; Caramel as color material; Aspartame and Acesulfame as sugar substitutes. Analyzing the placebo juice using colorimetric assay verified that the juice has no polyphenols. During placebo juice preparation, comparisons tests of flavor between placebo juice and Pomegranate Juice were performed by two independent testers to verify that placebo and Pomegranate Juice had a similar taste.

\section{Outcomes}

The primary endpoints were traditional CV risk factors including: number of antihypertensive drugs taken at the end of follow up compared to study initiation, mean SBP, DBP and PP, as well as lipid profile (mean levels of TG, HDL, LDL, total cholesterol).

Secondary outcome was incidence of CVD. CVD events as a composite variable, consisted of hospitalizations due to acute myocardial infarction (MI) (fatal and non fatal); ischemic stroke; new events of peripheral vascular disease (excluding the arterio-venous fistula) and unstable angina. Non fatal MI was defined as the presence of at least two of the following criteria: chest pain of typical duration and intensity, and/or increased cardiac enzymes and diagnostic electrocardiogram changes. Fatal MI was defined as a death occurring within $24 \mathrm{~h}$ from hospital admission. Death occurring outside hospital for which no other cause was assigned was regarded as sudden death and was included in the definition of CVD event.

\section{Statistics}

Data analysis was done with SPSS statistical analysis software. Continuous data are reported as mean \pm SD. The t-test for independent samples, or Mann Whitney test when appropriate, were used to detect differences in continuous variables between treatment groups. Frequency counts were calculated for categorical data. Differences in these variables were assessed by Chi Square Tests. In cases when expected values were lower than necessary, Fisher Exact Test was used. In order to study the time effect of PJ/Placebo on hypertention and lipid profile, repeated measures and Bonferroni post hoc analyses were done separately in each of the groups. In order to retain the intention to treat analysis we used the last observation carried forward method for missing data.

Survival curve comparing the effect of treatment on CVD events was calculated by the Kaplan-Meier method using the intention-to-treat (ITT) principle.

All statistical tests were two-sided, and statistical significance was defined as $\mathrm{P}<0.05$.

\section{Results}

\section{Characteristics of study population}

Characteristics of the 101 patients are described in our previous paper [18]. Briefly, the two studied groups were similar at study initiation in demographics, co-morbidities, number of drugs and biochemical characteristics. The study population's mean age was $66.5 \pm 11.8$ years and 54.5\% were males. Median follow-up time for the two groups was 12 months (range $0.25-12.6$ months). The total dropout rate was $33.7 \%$, insignificantly higher among PJ group $(37.8 \%)$ as compared to the placebo group (25.7\%). Patients who withdrew from the study did not differ from those who remained in their demographic, co-morbidy, treatment and biochemical characteristics. Furthermore, in spite of the $33.7 \%$ dropout rate, it should be noted that the baseline characteristics of patients who survived until the end of the study were similar at study initiation, indicating retained randomization success. Adverse events such as stomach upset or other GI-related effects were not demonstrated.

\section{Primary outcome}

The distribution of the number of anti-hypertensive medications used at study entry was similar between the two studied groups (mean $1.5 \pm 1.2$ drugs among PJ compared to $1.1 \pm 1.6$ drugs among placebo group, $\mathrm{p}=0.09$ ). After one year of intervention a significant $(\mathrm{P}=0.01)$ change in this distribution, as an ordinal variable (decrease, increase and no change) was noticed between the two group. The number of antihypertensive drugs decreased in $22.7 \%$ of the PJ patients, compared to $8.6 \%$ in the placebo group, while an increase was documented in $10.6 \%$ of the PJ patients compared to $31.4 \%$ in the placebo group (Figure 1). As shown in Table 1, among patients for whom the number of antihypertensive drugs did not change $(66.7 \%$ $(\mathrm{N}=44)$ and $60 \%(\mathrm{~N}=21)$ of patients in the PJ and placebo group, respectively), treatment with PJ was associated with a significant time response reduction in SBP and 


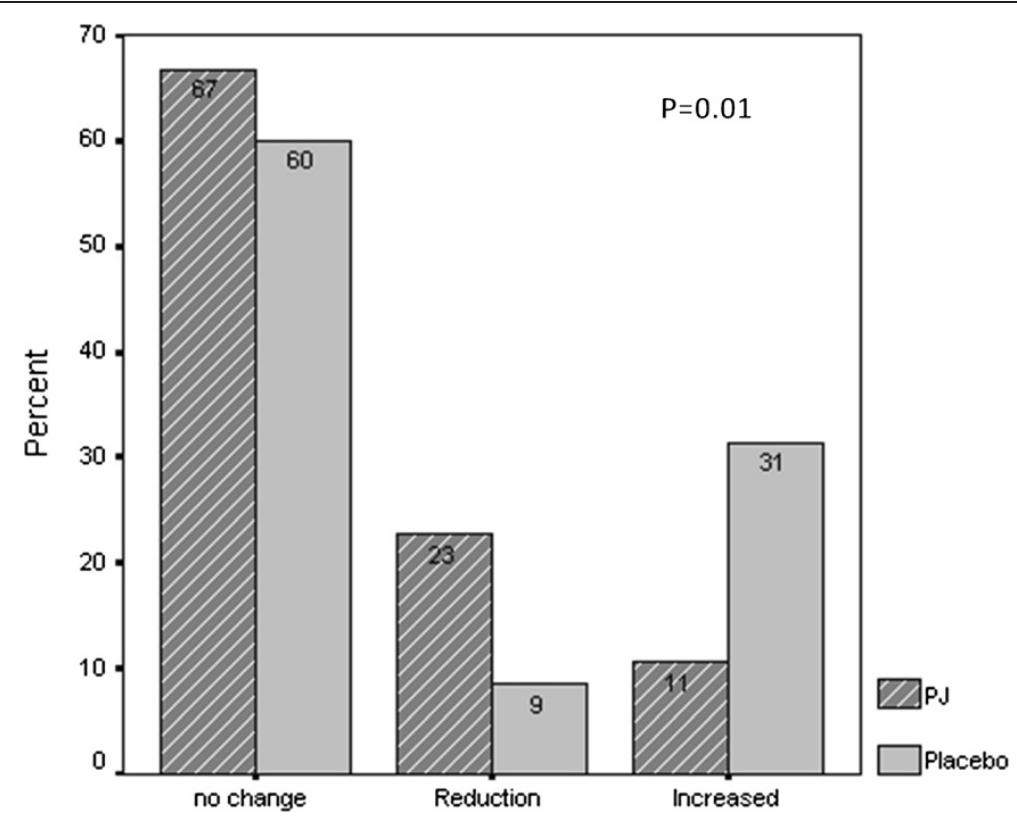

Figure 1 Changes in the number of anti HTN drugs after 12 months of intervention.

PP. Mean SBP, among patients in the PJ group was reduced after one year of intervention by $6.8 \%$ (Mean delta $9.8 \mathrm{~mm} \mathrm{Hg}$, P.V of paired T-test $=0.01$ ), while pulse pressure was reduced by $8.8 \%$ (Mean delta $6.6 \mathrm{~mm} \mathrm{Hg}, \mathrm{P} . \mathrm{V}$ of paired $\mathrm{T}$-test $=0.004)$. No significant changes were demonstrated among the placebo group. According to
Bonferroni test, the SBP at 12 months' intervention was significantly lower than the level at study initiation and at 3 months' intervention.

Among patients with baseline SBP $\geq 140 \mathrm{~mm} \mathrm{Hg}(\mathrm{N}=$ 26 for PJ group, $\mathrm{N}=5$ for placebo group) the significant effect of PJ consumption on blood pressure reduction

Table 1 SBP, DBP and PP during the study period by treatment group, repeated measures and Bonferroni post hoc analysis, using last observation carried forward method for missing data

\begin{tabular}{|c|c|c|c|c|c|c|c|c|}
\hline & \multirow[b]{2}{*}{ Time } & \multicolumn{3}{|l|}{ PJ } & \multicolumn{3}{|c|}{ Placebo } & \multirow{2}{*}{$\begin{array}{l}P^{* * * * *} \text { for PJ vs. } \\
\text { placebo at zero } \\
\text { time and at } 12 \mathrm{~m}\end{array}$} \\
\hline & & $\mathrm{N}$ & $\begin{array}{l}\text { Mean } \pm \text { SE } \\
(\mathrm{mm} \mathrm{Hg})\end{array}$ & $P$ for trend & $\mathrm{N}$ & $\begin{array}{l}\text { Mean } \pm \text { SE } \\
(\mathrm{mm} \mathrm{Hg})\end{array}$ & $P$ for trend & \\
\hline \multirow[t]{4}{*}{$\mathrm{SBP}^{\dagger}$} & 0 & 44 & $145.6 \pm 21.9$ & 0.001 & 21 & $129.6 \pm 27.2$ & 0.15 & 0.28 \\
\hline & $3 \mathrm{M}$ & & $143.6 \pm 23.9$ & & & $131.2 \pm 25.5$ & & \\
\hline & $6 \mathrm{M}$ & & $141.7 \pm 20.4$ & & & $138.8 \pm 24.8$ & & \\
\hline & $12 \mathrm{M}$ & & $135.7 \pm 21.3^{*}$ & & & $135.6 \pm 27.7$ & & 0.96 \\
\hline \multirow{4}{*}{$\begin{array}{l}\mathrm{SBP} \geq 140 \text { at } \\
\text { study initiation }\end{array}$} & 0 & 26 & $157.8 \pm 12.9$ & $<0.001$ & 5 & $162.0 \pm 10.6$ & 0.42 & 0.54 \\
\hline & $3 M$ & & $153.2 \pm 18.8$ & & & $157.1 \pm 13.9$ & & \\
\hline & $6 \mathrm{M}$ & & $149.5 \pm 14.8^{* *}$ & & & $156.2 \pm 17.6$ & & \\
\hline & $12 \mathrm{M}$ & & $144.0 \pm 16.9^{*}$ & & & $157.8 \pm 10.3$ & & 0.12 \\
\hline \multirow[t]{4}{*}{$\mathrm{DBP}^{\dagger+}$} & 0 & 44 & $70.9 \pm 11.4$ & 0.09 & 21 & $62.0 \pm 15.7$ & 0.46 & 0.09 \\
\hline & $3 \mathrm{M}$ & & $70.6 \pm 12.3$ & & & $64.5 \pm 13.5$ & & \\
\hline & $6 \mathrm{M}$ & & $69.6 \pm 12.5$ & & & $61.5 \pm 16.5$ & & \\
\hline & $12 \mathrm{M}$ & & $67.7 \pm 13.8$ & & & $63.8 \pm 20.4$ & & 0.38 \\
\hline \multirow[t]{4}{*}{$\mathrm{PP}^{+t+}$} & 0 & 44 & $74.6 \pm 19.5$ & 0.04 & 21 & $64.3 \pm 16.1$ & 0.02 & 0.24 \\
\hline & $3 \mathrm{M}$ & & $72.9 \pm 20.0$ & & & $66.5 \pm 19.2$ & & \\
\hline & $6 \mathrm{M}$ & & $72.1 \pm 17.0$ & & & $77.3 \pm 14.5^{* * * *}$ & & \\
\hline & $12 \mathrm{M}$ & & $68.0 \pm 16.6^{* * *}$ & & & $68.8 \pm 14.2$ & & 0.84 \\
\hline
\end{tabular}

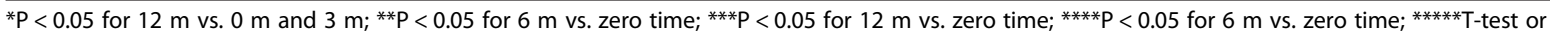
Mann-whitny $U$ test; $\uparrow$ Systolic blood pressure; ††Diastolic blood pressure; $\uparrow+\uparrow$ Pulse pressure. 
occurred earlier (after $6 \mathrm{~m}$ of intervention) and was more pronounced: SBP was reduced by $5.3 \%$ after $6 \mathrm{~m}$, and by $8.7 \%$ after $12 \mathrm{~m}$ of intervention (Table 1 ). No changes were observed in DBP in both groups.

Among patients in the placebo group a significantly increased PP was demonstrated. PJ consumption not only attenuated this increase but also significantly decreased it (Table 1).

Normal values of LDL and total cholesterol were demonstrated for the two study groups (Table 2). Treatment with PJ was not associated with significant changes in the above parameters. However, it was associated with significant time response improvements in HDL and TG levels, more evidently among patients with a pathologic level of these parameters (Table 2). Among patients with abnormal levels, following 12 months of intervention, levels of HDL were significantly higher while levels of TG significantly decreased among PJ patients as compared to the placebo subjects (Table 2).

\section{Secondary outcomes \\ CVD events}

The CVD incidence rates by group allocation, rate ratios and attributable risks are shown in Table 3. As seen, PJ intake, as compared to Placebo intake, was associated with $39 \%$ and $46 \%$ incidence rate reduction of the first and second CVD events, respectively. In addition, the reduction of the first event, as compared to the second event, was more pronounced in the PJ group (5.4 times lower incidence rate), as compared to the placebo group (4.7 times lower rate). Due to a small number of events, the difference between the two groups, as expressed by the Kaplan-Meier survival analysis, did not reach statistical significance (Figure 2).

\section{Discussion}

In the present study we demonstrated that intake of polyphenols-rich commercial PJ with anti-atherogenicity properties [13], improved the levels of SBP, PP, TG, and

Table 2 Lipid profile during the study period by treatment group, repeated measures and Bonferroni post hoc analysis, using last observation carried forward method for missing data

\begin{tabular}{|c|c|c|c|c|c|c|c|c|}
\hline & & \multicolumn{3}{|l|}{ PJ } & \multicolumn{3}{|c|}{ Placebo } & \multirow{2}{*}{$\begin{array}{l}P^{* * *} \text { for } P J \text { vs. } \\
\text { placebo at } 12 \mathrm{~m}\end{array}$} \\
\hline & & $\mathbf{N}$ & Mean \pm SD & $P$ for trend & $\mathbf{N}$ & Mean \pm SD & $P$ for trend & \\
\hline \multirow[t]{4}{*}{$\mathrm{LDL} \mathrm{mg} / \mathrm{dl}$} & 0 & 66 & $95.1 \pm 31.5$ & 0.07 & 35 & $95.8 \pm 28.9$ & 0.38 & 0.85 \\
\hline & $3 M$ & & $98.9 \pm 35.6$ & & & $98.0 \pm 28.1$ & & \\
\hline & $6 M$ & & $95.4 \pm 32.4$ & & & $91.8 \pm 25.1$ & & \\
\hline & $12 \mathrm{M}$ & & $100.0 \pm 33.1$ & & & $94.3 \pm 27.2$ & & 0.39 \\
\hline \multirow[t]{4}{*}{ Total cholesterol mg/dl } & 0 & 66 & $164.8 \pm 37.1$ & 0.27 & 35 & $163.5 \pm 33.4$ & 0.18 & 0.86 \\
\hline & $3 M$ & & $167.7 \pm 40.7$ & & & $171.7 \pm 35.1$ & & \\
\hline & $6 \mathrm{M}$ & & $163.4 \pm 38.9$ & & & $164.8 \pm 38.0$ & & \\
\hline & $12 \mathrm{M}$ & & $167.3 \pm 43.5$ & & & $165.1 \pm 35.8$ & & 0.79 \\
\hline \multirow[t]{4}{*}{ HDL mg/dl } & 0 & 66 & $33.1 \pm 9.5^{*}$ & $<0.001$ & 35 & $36.6 \pm 10.7$ & 0.18 & 0.10 \\
\hline & $3 M$ & & $37.2 \pm 11.3$ & & & $37.0 \pm 11.7$ & & \\
\hline & $6 M$ & & $36.6 \pm 10.9$ & & & $37.3 \pm 12.9$ & & \\
\hline & $12 \mathrm{M}$ & & $36.8 \pm 10.8$ & & & $34.3 \pm 15.4$ & & 0.40 \\
\hline \multirow{4}{*}{$\begin{array}{l}\mathrm{HDL} \leq 40 \mathrm{mg} / \mathrm{dl} \\
\text { at study initiation }\end{array}$} & 0 & 54 & $29.7 \pm 5.7^{*}$ & $<0.001$ & 23 & $30.2 \pm 5.6$ & 0.09 & 0.84 \\
\hline & $3 M$ & & $33.8 \pm 7.8$ & & & $31.2 \pm 7.6$ & & \\
\hline & $6 M$ & & $33.6 \pm 7.7$ & & & $31.8 \pm 10.2$ & & \\
\hline & $12 \mathrm{M}$ & & $33.6 \pm 7.5$ & & & $27.6 \pm 11.6$ & & 0.03 \\
\hline \multirow[t]{4}{*}{$\mathrm{TG} \mathrm{mg} / \mathrm{dl}$} & 0 & 66 & $183.6 \pm 101.6^{* *}$ & 0.05 & 35 & $176.7 \pm 86.7$ & 0.04 & 0.73 \\
\hline & $3 M$ & & $175.7 \pm 90.8$ & & & $192.9 \pm 100.7$ & & \\
\hline & $6 \mathrm{M}$ & & $174.0 \pm 89.8$ & & & $193.6 \pm 90.5$ & & \\
\hline & $12 \mathrm{M}$ & & $167.3 \pm 86.3$ & & & $206.1 \pm 109.4$ & & 0.05 \\
\hline \multirow{4}{*}{$\begin{array}{l}\mathrm{TG} \geq 200 \mathrm{mg} / \mathrm{dl} \\
\text { at study initiation }\end{array}$} & 0 & 20 & $310.1 \pm 87.8^{* *}$ & 0.02 & 10 & $288.1 \pm 71.9$ & 0.15 & 0.13 \\
\hline & $3 M$ & & $267.3 \pm 86.6$ & & & $318.2 \pm 96.6$ & & \\
\hline & $6 \mathrm{M}$ & & $248.7 \pm 100.5$ & & & $301.5 \pm 78.2$ & & \\
\hline & $12 \mathrm{M}$ & & $237.4 \pm 102.5$ & & & $320.4 \pm 56.8$ & & 0.008 \\
\hline
\end{tabular}


Table 3 Effect of PJ intake on the incidence rate, rate ratio and attributable risk of first and second CVD events

\begin{tabular}{|c|c|c|c|c|c|}
\hline CV Events & Group & Incidence rate & Rate ratio & P.V (95\% Cl) & Attribute risk \\
\hline \multirow[t]{2}{*}{ First event } & PJ & $\frac{10}{648.24 P M} \times 1000=15.42 / 1000 P M$ & \multirow[t]{2}{*}{0.61} & \multirow[t]{2}{*}{$0.30(0.25-1.5)$} & $25.03 / 1000 \mathrm{PM}^{-15.42} / 1000 \mathrm{PM}$ \\
\hline & Placebo & $\frac{9}{359.57 P M} \times 1000=25.03 / 1000 P M$ & & & \\
\hline \multirow[t]{2}{*}{ Second event } & PJ & $\frac{2}{701.42 P M} \times 1000=2.85 / 1000 P M$ & \multirow[t]{2}{*}{0.54} & \multirow[t]{2}{*}{$0.55(0.08-3.9)$} & \multirow[t]{2}{*}{$\frac{5.34 / 1000 \mathrm{PM}-2.85 / 1000 \mathrm{PM}}{5.34 / 1000 \mathrm{PM}} \times 100=46.3 \%$} \\
\hline & Placebo & $\frac{2}{376.46 P M} \times 1000=5.31 / 1000 P M$ & & & \\
\hline
\end{tabular}

HDL among HD patients. These effects were more notable among patients with higher levels of SBP and TG and among subjects with lower levels of HDL. PJ intake was also found to be related to the decrease in the number of anti hypertensive drugs, highlighting its beneficial effect on blood pressure level. It must be emphasized that when comparing the two treatment groups HDL and TG were the only parameters who demonstrated significant differences, which might be explained for both factors by an improvement in the PJ group and impairment in the placebo group. Furthermore, PJ had no demonstrable effect on total cholesterol and LDL levels, probably due to their normal levels at study entry. Our results are in agreement with studies conducted in other chronic populations, demonstrating reduction in SBP [13] and improvement in lipid profiles among patients who consumed PJ [16]. Our study demonstrated for the first time the anti-hypertensive and anti- hyperlipidemic properties of PJ among HD patients.
It is widely accepted that hypertension and dyslipidemia are contributors to the atherosclerotic process, as well as independent predictors of CVD in CKD and dialysis patients [1]. As such, our results in the current and previous study [18] indicate the potentially important cardioprotective role of PJ. In spite of the above we did not succeed to show a significant reduction in the incidence of CVD, probably due to the longer intervention period needed to demonstrate a statistically significant effect.

The mechanism by which PJ consumption reduced SBP may relate to its ability to decrease ACE activity (secondary to its antioxidant properties) or may be due to a direct effect on serum ACE activity [13]. Furthermore, as reactive oxygen species (ROS) contribute to endothelium dependent contraction and to increased vascular resistance, the antioxidative effects by PJ, as we previously demonstrated [18], can possibly restore endothelial function and hence decrease blood pressure $[13,22]$. SBP reduction may also be a result of PJ's ability
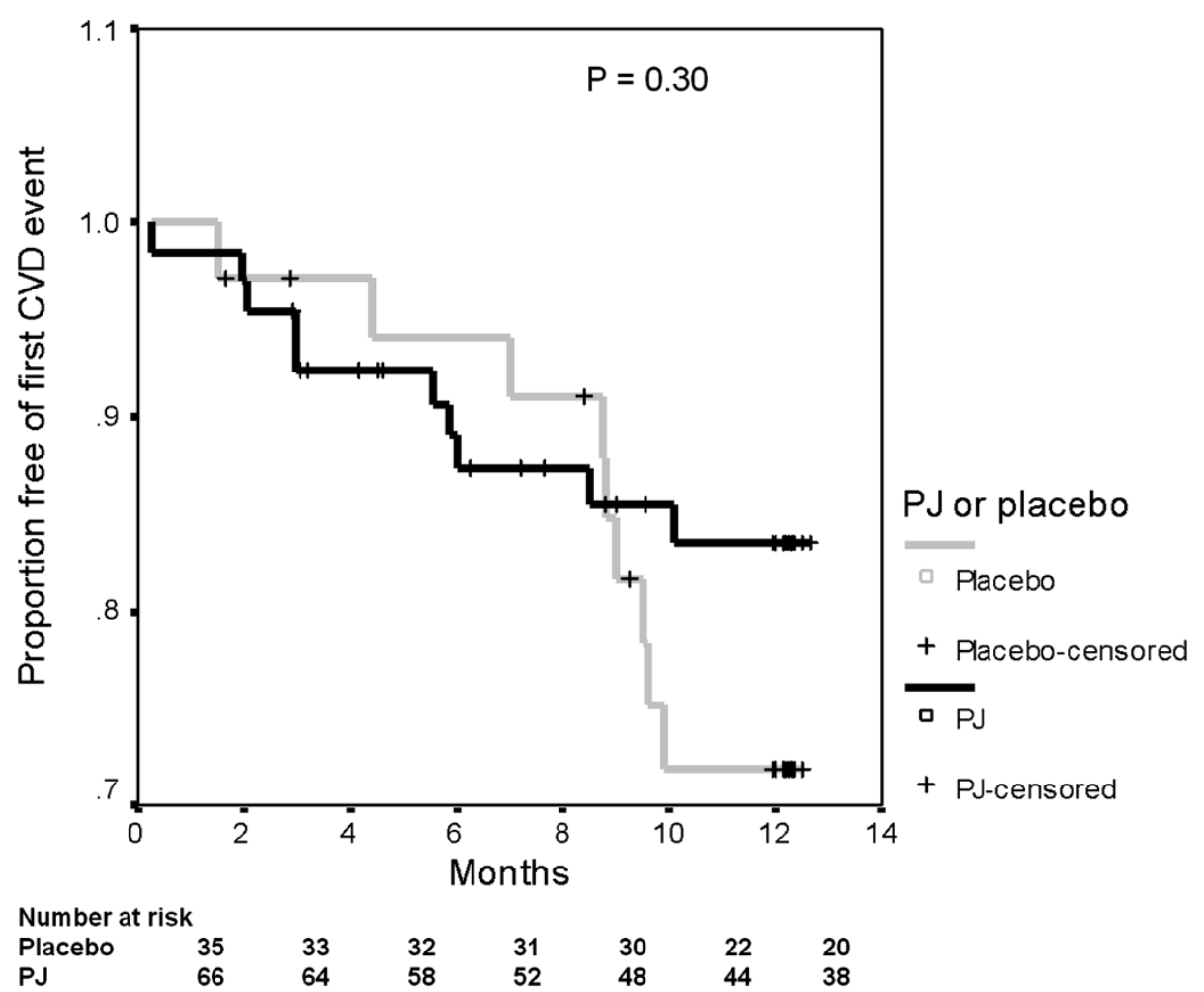

Figure 2 Kaplan-Meier survival curves following first CVD event. 
to protect nitric oxide against oxidative destruction [23] and to enhance the nitric oxide synthase bioactivity [24]. In contrast to SBP reduction, the mechanism underlying the anti- hyperlipidemic effect of PJ is unclear. Both an inhibition of intestinal absorption, as well as accelerated clearance of plasma triglycerides lipid may account for the observed hypolipemic action of PJ [25]. The beneficial effects of PJ on HDL and TGs could be mediated by its ability to induce increment in paraoxonase 1 [26], protecting HDL from oxidation $[27,28]$, and in paraoxonase 2 [29], influencing TG accumulation by macrophages as well their TG synthesis [30].

Important questions related to interpretation of our above results are whether the associations found are real and causal. Taking into consideration the uncompromising methodology described, there is no reason to believe that biases have been introduced. As for causality, all Hill's criteria were met: the clinical trial methodology assured temporal relationship issues; the protective associations between PJ exposure and cardiovascular events were found strong; our findings regarding the association between polyphenols- rich juice and its anti-hypertensive and hypolipemic properties were consistently described across other studies of divergent designs and populations $[13,16,25]$; a time response relationship was demonstrated between the intervention duration and outcomes; and there is a possible biological explanation for the relationships noticed between PJ intake and the demonstrated results (described above). Nevertheless, some methodological limitations should be mentioned: first, this study was a small single center trial, which may limit its external validity (generalizability); though the sample size was adequate to examine all study assumptions. In addition, data collection by one investigator in one trial center assured high quality of data collected. Second, there is no data regarding the pharmacokinetics of polyphenols gastrointestinal absorption among HD patients. However, the effect on SBP and lipid profile was demonstrated only among the PJ group, supporting the notion of a PJ effect.

\section{Conclusions}

The significant beneficial effect of PJ intake on SBP, PP and lipid profile among HD patients, in addition to its beneficial effect on oxidative stress and inflammation, suggests that constant PJ consumption can offer wide protection against cardiovascular events - the main cause of morbidity and mortality among HD patients.

Furthermore, as controlled consumption of PJ has been shown to lower morbidities in HD patients it is expected to reduce costs associated with those patients' care. Further multi centered clinical studies are needed to substantiate our findings that while directly improving patients' quality of life, PJ can also significantly reduce health expenditure. Such studies might well influence future policy makers to include PJ as part of state-funded standard care for HD patients.

\section{Abbreviations}

CVD: Cardiovascular disease; HD: Hemodialysis; PJ: Pomegranate juice; CV: Cardiovascular; TG: Triglycerides; HDL: High density lipoprotein; LDL: Low density lipoprotein; RRT: Renal replacement therapy; CKD: Chronic kidney disease; ACE: Angiotensin converting enzyme; RAAS: Renin angiotensin aldosterone system; IMT: Intima-media thickness; BP: Blood pressure; SBP: Systolic blood pressure; DBP: Diastolic blood pressure; PP: Pulse pressure; MI: Myocardial infarction; ITT: Intention-to-treat.

\section{Competing interests}

The authors declare that they have no competing interests.

\section{Authors' contributions}

All authors have contributed to the conception and design of the work. We had full access to all of the data in this study, and we take complete responsibility for the integrity of the data and the accuracy of the data analysis. We declare that we have seen and approved the final version.

\section{Acknowledgments}

This study was supported by grant no. 6186 from the Chief Scientist Office of the Ministry of Health, Israel; grant no. 2012255 from Jess \& Midred Fisher Family Cardiology Research Fund, Office of the executive vice president for research, Technion, Israel; and by Iscar Ltd.

\section{Author details}

'Quality Assurance Department, Western Galilee Hospital - Nahariya, Nahariya, Israel. ${ }^{2}$ Nephrology Department, Western Galilee Hospital Nahariya, Nahariya, Israel. ${ }^{3}$ Faculty of Medicine in the Galilee, Bar Ilan University, Safed, Israel. ${ }^{4}$ Clinical Microbiology Lab and Eliachar Research Lab, Western Galilee Hospital - Nahariya, Nahariya, Israel. ${ }^{5}$ School of Public Health, Faculty of Social Welfare \& Health Sciences, University of Haifa, Haifa, Israel.

Received: 22 July 2013 Accepted: 17 February 2014

Published: 4 March 2014

\section{References}

1. Kundhal K, Lok C: Clinical epidemiology of cardiovascular disease in chronic kidney disease. Nephron Clin Pract 2005, 101:c47-c52.

2. Collins AJ, Foley R, Herzog C, Chavers B, Gilbertson D, Ishani A, Kasiske B, Liu J, Mau LW, McBean M, Murray A, St Peter W, Xue J, Fan Q, Guo H, Li Q, Li S, Li S, Peng Y, Qiu Y, Roberts T, Skeans M, Snyder J, Solid C, Wang C, Weinhandl E, Zaun D, Zhang R, Arko C, Chen SC, et al: Excerpts from the United State renal data system 2007 annual data report. Am J Kidney Dis 2008, 51:S1-320.

3. Maheshwari N, Ansari M, Darshana M, Lal K, Ahmed K: Pattern of lipid profile in patients on maintenance hemodialysis. Saudi J Kidney Dis Transplant 2010, 21:565-570.

4. Herspink $H$, Ninomiya $T$, Zoungas $S$, de- Zeeuw $D$, Grobbee $D$, Jardine $M$, Gallagher M, Roberts M, Cass A, Neal B, Perkovic V: Effect of lowering blood pressure on cardiovascular events and mortality in patients on dialysis: a systematic review and meta-analysis of randomised controlled trials. Lancet 2009, 373:1009-1015.

5. Prichard S: Impact of dyslipidemia in end-stage renal disease. J Am Soc Nephrol 2003, 14:S315-S320.

6. Deighan C, Caslake M, McConnell M, Boulton-Jones J, Packard C: Atherogenic lipoprotein phenotype in end-stage renal failure: origin and extent of small dense low density lipoprotein formation. Am J Kidney Dis 2000, 35:852-862.

7. Perkovic V, Ninomiya T, Arima H, Gallagher M, Jardine M, Cass A, Neal B, Macmahon S, Chalmers J: Chronic kidney disease, cardiovascular events, and the effects of perindopril-based blood pressure lowring: data from the PROGRESS study. J Am Soc Nephrol 2007, 18:2766-2772.

8. Bloob pressure lowering treatment trialists' collaboration: Effects of ACE inhibitors, calcium antagonists, and other blood-pressure-lowering drugs: results of prospectively designed overviews of randomised trials. Lancet 2000, 356:1955-1964.

9. Fabbri G, Maggioni A: Cardiovascular risk reduction: what do recent trials with rosuvastatin tell us? Adv Ther 2009, 26:469-487. 
10. Seliger S, Weiss N, Gillen D, Kestenbaum B, Ball A, Sherrard D, Stehman-Breen C: HMG-CoA reductase inhibitors are associated with reduced mortality in ESRD patients. Kidney Int 2002, 61:297-304.

11. Bors W, Heller W, Michel C, Saran M: Flavonoids as antioxidants: determination of radical-scavenging efficiencies. Methods Enzymol 1990, 186:343-355.

12. Cook N, Samman S: Flavonoids- chemistry, metabolism, cardio protective effects and dietary sources. J Nutr Biochem 1996, 7:66-76.

13. Aviram M, Dornfeld L: Pomegranate juice consumption inhibits serum angiotensin converting enzyme activity and reduces systolic blood pressure. Atherosclerosis 2001, 158:195-198.

14. Aviram M, Rosenblat M, Gaitini D, Nitecki S, Hoffman A, Dornfeld L, Volkova $\mathrm{N}$, Presser D, Attias J, Liker H, Hayek T: Pomegranate juice consumption for 3 years by patients with carotid artery stenosis reduces common carotid intima-media thickness, blood pressure and LDL oxidation. Clin Nutr 2004, 23:423-433.

15. Summer M, Elliott-Eller M, Weidner G, Daubenmier J, Chew M, Marlin R, Raisin C, Ornish D: Effects of pomegranate juice consumption on myocardial perfusion in patients with coronary heart disease. J Cardiol 2005, 96:810-814.

16. Esmaillzadeh A, Tahbaz F, Gaieni I, Alavi-Majd H, Azadbakht L: Concentrated pomegranate juice improves lipid profiles in diabetic patients with hyperlipidemia. J Med Food 2004, 7:305-308.

17. Rosenblat M, Hayek T, Aviram M: Anti-oxidative effects of pomegranate juice (PJ) consumption by diabetic patients on serum and on macrophages. Atherosclerosis 2006, 187:363-371.

18. Shema-Didi L, Sela S, Ore L, Shapiro G, Geron R, Moshe G, Kristal B: One year of pomegranate juice intake decreases oxidative stress, inflammation and incidence of infections in hemodialysis patients: a randomized placebo-controlled trial. Free Radic Biol Med 2012, 53:297-304.

19. Dumville J, Hahn S, Miles J, Torgerson D: The use of unequal randomisation ratios in clinical trials: a review. Contemp Clin Trials 2006, 27:1-12.

20. Singleton $V$, Rossi J: Colorimetry of total polyphenols with phosphomolybdic phosphotungstic acid reagents. Am J Emology Viticulture 1965, 16:144-158.

21. Ben Nasr C, Ayed N, Metche M: Quantitative determination of the polyphenols content pg pomegranate peel. Z Lebensum Unters Forsch 1996, 203:374-378.

22. Kitiyakara C, Wilcox C: Antioxidants for hypertension. Curr Opin Nephrol Hypertens 1998, 7:531-538.

23. Ignarro L, Byrns R, Sumi D, Nigris F, Napoli C: Pomegranate juice protects nitric oxide against oxidative destruction and enhances the biological actions of nitric oxide. Nitric Oxide 2006, 15:93-102.

24. Nigris F, Williams-Ignarro S, Botti C, Sica V, Ignarro L, Napoli C: Pomegranate juice reduces oxidized low density lipoprotein downregulation of endothelial nitric oxide synthase in human coronary endothelial cell. Nitric Oxide 2006, 15:259-263.

25. Castilla P, Echarri R, Davalos A, Cerrato F, Ortega H, Teruel J, Lucas M, Gomez-Coronado D, Ortuno J, Lasuncion M: Concentrated red grape juice exerts antioxidant, hypolipoidemic, and antiinflammatory effects in both hemodialysis patients and healthy subjects. Am J Clin Nutr 2006, 84:252-262

26. Rock W, Rosenblat M, Miller-Lotan R, Levy A, Elias M, Aviram M: Consumption pf wonderful variety pomegranate juice and extract by diabetic patients increases paraoxonase 1 associated with high density lipoprotein and stimulates its catalytic activities. J Agric Food Chem 2008, 56:8704-8713.

27. Aviram M, Rosenblat M, Bisgaier C, Newton R, Primo-Parmo S, La Du B: Paroxonase inhibits High-density lipoprotein oxidation and preserves its functions. J Clin Invest 1998, 101:1581-1590.

28. Aviram M, Dornfeld L, Rosenblat M, Volkova N, Kaplan M, Coleman R, Hayek T, Presser D, Fuhrman B: Pomegranate juice consumption reduces oxidative stress, atherogenic modifications to LDL, and platelet aggregation: studies in humans and in atherosclerotic apolipoprotein E-deficient mice. Am J Clin Nutr 2000, 71:1062-1076.
29. Shiner M, Fuhrman B, Aviram M: Macrophage paraoxonase 2 (PON2) expression is up-regulated by pomegranate juice phenolic anti-oxidants via PPAR and AP-1 pathway activation. Atherosclerosis 2007, 195:313-321.

30. Rosenblat M, Coleman R, Reddy S, Aviram M: Paraoxonase 2 (PON2) attenuates macrophage triglyceride accumulation via inhibition of diacyglycerol acyltransferase 1, the rate limiting enzyme in triglycerides biosynthesis. J Lipid Res 2009, 50:870-879.

doi:10.1186/1475-2891-13-18

Cite this article as: Shema-Didi et al.: Does Pomegranate intake attenuate cardiovascular risk factors in hemodialysis patients? Nutrition Journal 2014 13:18.

\section{Submit your next manuscript to BioMed Central and take full advantage of:}

- Convenient online submission

- Thorough peer review

- No space constraints or color figure charges

- Immediate publication on acceptance

- Inclusion in PubMed, CAS, Scopus and Google Scholar

- Research which is freely available for redistribution

Submit your manuscript at www.biomedcentral.com/submit
C Biomed Central 